\title{
Influencia del entorno sobre el riesgo asociado a la Banca Universal Venezolana
}

\author{
Castellano Montiel, Alberto G.*
}

\section{Resumen}

En los últimos años, la situación económica y política del país ha influido enormemente en la situación financiera de muchas empresas, ésto se observa en el gran número de empresas que han cerrado sus puertas desde 1999. A dicha situación no ha escapado el sector financiero el cual ha experimentado el fuerte impacto que estos cambios coyunturales han tenido en la economía del país. Por ello este trabajo pretende identificar la influencia del entorno político económico en el país sobre la situación financiera de la banca universal venezolana. En este sentido se realiza un estudio descriptivo sobre la base de coeficientes de determinación, elaborando para ello un modelo de regresión lineal, utilizando técnicas de evaluación como el análisis discriminante y la metodología de la superintendencia de bancos y otras instituciones financieras, para examinar el riesgo que presenta el sector y cómo ha influido el entorno en esta situación. Para su análisis se asumirá una relación causal entre las variables entorno y situación financiera, realizando un trabajo empírico basado en datos de la banca comercial y universal e información financiera. Todo ello lleva a elaborar conclusiones sobre aspectos tantos internos como externos que afectan al sector bancario específicamente la banca universal venezolana, teniendo como resultado la existencia de un proceso de elásticidad de la situación financiera del sector con respecto al entorno político económico, ocasionando un incremento del riesgo sistemático del sector con respecto a su entorno. Además de la relación existente entre los resultados del análisis discriminante con los resultados de la metodología de la superintendencia de bancos y otras instituciones financieras.

Palabras clave: Riesgo de mercado, Banca Universal, análisis discriminante, Venezuela, riesgo país.

Recibido: 00-07-27 . Aceptado: 01-06-18

Economista, Magister en Gerencia de Empresas, Mención Gerencia Financiera, Cursante del Doctorado de Ciencias Económicas, Investigador- Docente del Instituto de Investigaciones, Facultad de Ciencias Económicas y Sociales, La Universidad del Zulia, Dirección: Avenida Ziruma con Prolongación Circunvalación No 2, Núcleo Humanístico. E. Mail: albercas@luz.ve. Telfax (58261) 7596584. 


\section{The Influence of Environment on Risk Associated with the Universal Venezuelan Bank}

\section{Abstract}

Over the last few years, the economic and political situation of the country has greatly influenced the financial situation of many businesses, and this is observed in the great number of firms that have shut down since 1999. The financial sector does not escape this situation and reflects the strong impact of these changes in the economy of the country. This paper proposes to identify the influence of the political-economic environment in reference to the financial situation of the universal Venezuelan bank. To do this a descriptive study of determinant coefficients, elaborated by a linear regression model was made utilizing evaluation techniques such as discriminate analysis, as well as the methodology used by the Superintendence of Banks and other financial institutions to examine risk levels present in the financial sector and to evaluate how they have affected the environment. In the analysis, a causal relationship was assumed between the environmental variables and the financial situation, and empirical work based on data from the commercial and universal bank sectors and financial information was carried out. All this lead to the elaboration of conclusions as to both internal and external aspects that affect the banking community, and specifically the universal Venezuelan bank. The results seem to indicate the existence of a process of elasticity in the financial situation in reference to the political-economic situation, stimulating an increase in the systematic risk of this sector in reference to the environment. There is a relation between the results of the discriminate analysis and the results of the methodology applied by the Superintendence of Banks and other institutions.

Key words: Market risk, universal bank, discriminate analysis, Venezuela, risk, country.

\section{Introducción}

En el transcurso de los años sesenta (60) y setenta (70), en Venezuela era desconocida la presencia de riesgo, de hecho con la inflación girando alrededor de un porcentaje de cero, con un signo monetario (Bolívar) estable y con una economía en general en crecimiento, era casi absurdo referirse al riesgo, aunque otros países manejaban todo lo concerniente al mismo. Todo ello generó un caldo de cultivo propicio para el funcionamiento del sistema bancario, cuya finalidad primordial se basaba en proveer financiamiento al sector empresarial, el cual se desenvolvía en condiciones favorables, debido en parte a los continuos subsidios recibidos del
Estado; reflejando la banca, en términos generales, niveles muy favorables de rentabilidad y de liquidez.

Cabe considerar por otra parte, que en la década de los ochenta Venezuela comienza un prolongado período de estancamiento económico con altos niveles de inflación (denominado estanflación), inducido fundamentalmente por una coyuntura internacional difícil; explicada por el debilitamiento de los precios del petróleo, la recesión en los países industrializados, la crisis del sistema financiero internacional a partir de 1982, y por los desaciertos de las políticas económicas aplicadas durante el decenio. Esta situación contrasta con el exitoso y estable crecimiento macroeconómico que había 
experimentado Venezuela durante el período de 1950 a 1980.

Es a partir de 1989 cuando debido a la grave situación económica del país, el gobierno adopta una concepción diferente en la aplicación de políticas económicas, orientándose al crecimiento hacia afuera, para ello ejecuta una serie de reformas estructurales, buscando corregir los desequilibrios macroeconómicos, teniendo un enorme impacto negativo so bre el sector empresarial y financiero; acostumbrados a un medio ambiente sobreprotegido y con las condiciones propicias, incursionando en un nuevo mundo donde prevalece el riesgo teniendo que tomar medidas para convivir con él y evitar los efectos desfavorables del entorno político económico. Las actuales condiciones económicas, se caracteriza básicamente por la devaluación de la moneda y altos niveles de estanflación, aunado al proceso de desaparición de las fronteras tanto a nivel político como económico en general, denominado globalización; dado el efecto mundial que han tenido la consecución de crisis locales como la mexicana y más recientemente la asiática y la rusa, ello incrementa el interés por el conocimiento acerca del riesgo; siendo hoy por hoy de uso común conceptos como: riesgo país, calificadora de riesgo, entre otros.

En este sentido, la banca nacional, los depositantes y los entes reguladores del Estado han tomado muy en serio la calificación de riesgo y la influencia del entorno, debido en parte a la crisis bancaria experimentada en 1994, la cual afectó a la economía del país.

\section{Consideraciones generales}

Para el estudio de la influencia del entorno en la banca universal venezolana se analizarán los distintos factores que inciden sobre el comportamiento financiero de la banca, teniendo como resultado una lista de tipos de riesgos.

\subsection{Diferentes líneas de riesgo}

Los diferentes factores que influyen y condicionan la evolución de los mercados y de las instituciones financieras en general, son los que contribuyen a los cambios generados en el negocio bancario; tradicionalmente la banca se ha dedicado a la intermediación financiera, pero poco a poco fue incrementando sus funciones aunado a la existencia de nuevos competidores bancarios y no bancarios (actuales y potenciales). La banca asume por sus propias funciones y operaciones, diferentes tipos de riesgos: de crédito, liquidez, de tipo de cambio, de tipo de interés, entre otros, pero en la actualidad existen nuevos enfoques sobre el riesgo, López y Sebastián (1998: 218221) determina siete tipos de riesgo, los cuales expresan lo siguiente:

Riesgo de crédito: se le denomina así a la probabilidad de que un prestatario (cliente activo) no devuelva el principal de su préstamo o crédito y no pague los intereses de acuerdo con lo estipulado en el contrato. Esta actividad constituye la principal fuente de ingresos para un banco, pero puede ser a su vez la causa determinante de la quiebra de un instituto financiero, dado que un prés- 
tamo puede aportar tantos ingresos a la cuenta de resultados, como puede ocasionar serios problemas de liquidez en caso de morosidad en el pago de los intereses y del principal.

Riesgo-país: se define el riesgopaís como la posibilidad de que el conjunto de prestatarios de una nación sean incapaces de cancelar los intereses y de devolver el principal de sus deudas a sus acreedores extranjeros.

Riesgo de liquidez: este tipo de riesgo refleja la posible pérdida en que puede incurrir una entidad que se ve obligada a vender activos o a contraer pasivos en condiciones desfavorables, para hacer frente a retiros o para satisfacer la demanda de solicitudes de crédito.

Riesgo de mercado: se refiere a la posibilidad de que el banco sufra una pérdida en un determinado período, debido a movimientos inesperados y adversos en los tipos de interés, y de cambio.

Riesgo tecnológico: es la exposición que tienen las instituciones financieras a pérdidas por posibles fallos en el sistema tecnológico, dado su peso dentro de la actividad bancaria.

Riesgo operativo: fallos ocurridos en el proceso de seguimiento y control de las posiciones asumidas; en general inadecuado control interno.

Riesgo legal: supone la realización de una pérdida debido a que una operación no puede ejecutarse por incapacidad de una de las partes para cumplir los compromisos contractuales asumidos, por no existir una formalización clara o no ajustarse al marco legal establecido.

Finalmente en el riesgo se puede identificar dos componentes uno siste- mático y otro no sistemático, en este sentido se expresa: "El riesgo sistemático es el que puede atribuirse a una cartera de inversiones bien diversificada y, por definición, no puede reducirse; el riesgo no sistemático puede reducirse, y hasta eliminarse, mediante la diversificación de la cartera" (Sabal, 1997: 3).

Tomando en cuenta el presente listado de tipos de riesgo, es de hacer notar que dada la complejidad del riesgo asociado a la banca universal por lo diverso de sus operaciones y por su naturaleza misma, en la presente investigación se estudia lo referente a riesgo de mercado y riesgo-país para una mejor comprensión del mismo y facilidad en el manejo de las variables.

En este sentido, para la consecución de esta investigación se entenderá como concepto de riesgo país al riesgo que se asocia a un país dado la aplicación de políticas económicas internas, que inciden sobre la confiabilidad de los inversionistas; igualmente se asumirá el concepto de riesgo de mercado citado anteriormente.

Para el análisis de las influencias del entorno se analiza el concepto de desintermediación financiera como condicionante para el incremento del riesgo el cual permite visualizar su posible desenvolvimiento.

\subsection{Desintermediación financiera}

Se entiende este proceso como: "la pérdida de presencia de los intermediarios financieros tradicionales entre las economías ahorradoras y las economías inversoras, debido a que otras institucio- 
nes, financieras o no, asumen parte creciente de esta presencia" (López y Sebastián, 1998: 22).

En este sentido, los ahorristas retiran sus depósitos de las instituciones financieras cuando la compra directa de valores financieros emitidos por familias, corporaciones y el gobierno, ofrece mayores niveles de rentabilidad; por ello, "el volumen de desintermediación depende en gran parte de las diferencias existentes entre las tasas de captación que ofrecen los intermediarios y aquellas a las que captan los prestatarios últimos como corporaciones y gobiernos" (Ramírez y Díaz, 1999: 396).

Al igual que las empresas y el sector gobierno que emiten títulos y valores obteniendo financiamiento a menor costo, los compradores de títulos también se benefician al obtener tasas con mayor rentabilidad; en pocas palabras, las empresas pagan un menor interés por endeudarse y los inversionistas una mayor rentabilidad por sus inversiones. El proceso descrito origi na un mercado más competitivo en número y calidad de productos y servicios ofrecidos, obviando en muchas ocasiones la acción de intermediarios financieros, sobre todo de la banca.

Para su estudio, Ramírez y Díaz, (1999: 398) identifican diferentes causas que originan la desintermediación financiera, junto a las características que lo identifican.

1. Las Innovaciones tecnológicas: es causante de desintermediación en la medida que garantiza comunicaciones más rápidas y eficaces; en este sentido han surgido transacciones en línea vía internet, cajeros automáti- cos entre otros, posibles de utilizar no sólo por el sector bancario.

2. El auge del mercado bursátil: presenta mayores facilidades para obtener recursos de capital y también para incrementar la liquidez de las acciones.

3. El fomento de mercados secundarios, que amplía las alternativas de los inversionistas institucionales.

4. La integración de los mercados internacionales: implica un aumento de los flujos externos al país, bien por vía de capitales o por préstamos.

5. La aparición de inversionistas institucionales: intensifica la competencia en el mercado financiero ofreciendo servicios que tradicionalmente le pertenecen a los bancos.

Las principales características observadas en el desarrollo del proceso de desintermediación financiera se enumeran a continuación:

1. Se genera una desinversión de parte de las empresas a través de la banca; encontrando en forma alternativa, los fondos para su financiamiento en el mercado de capitales, sin intermediación de la banca. Igualmente las empresas pueden colocar sus superávits de corto plazo nuevamente en el mercado de capitales sin intermediación de la banca.

2. Como consecuencia del proceso, se reducen los costos a través de la disminución del diferencial entre la tasa de interés activa y pasiva (spreads) al existir un ambiente de competitividad de diversos productos financieros e intermediarios financieros.

3. Diversificación del mercado de capitales: debido a la entrada de entidades no bancarias en el negocio de los 
préstamos e inversión, principalmente las Administradoras de Fondos de Pensiones, Compañías de Seguro y Fondos Mutuales.

Dentro de este orden de ideas, se pretende determinar el riesgo de mercado asociado a la banca universal venezolana, para observar la incidencia de los factores sobre el mismo, para ello se utilizará la metodología de la Superintendencia de Bancos y otras Instituciones financieras, la cual consiste en lo siguiente:

\subsection{Metodología para la clasificación de riesgo de la banca según su desempeño}

\section{La Superintendencia de Bancos y} Otras Instituciones Financieras (1998) basándose en criterios establecidos en convenios internacionales y tomando en cuenta las razones financieras que permiten delinear el perfil de una institución financiera, ha desarrollado esta metodología que permite la clasificación de riesgo de la banca Comercial y Universal del sistema venezolano, utilizando para ello las categorías de índices especificados en el modelo propuesto por el congreso de Basilea denominado CAMEL:

C: Evaluación del Patrimonio, A: Calidad de Activos, M: Gestión Administrativa,

E: Rentabilidad y L: Liquidez.

La calificación se basa en la definición de cada indicador y su evolución histórica. Para el análisis de cada una de estas categorías se determina un conjunto de indicadores financieros que resultan de relacionar dos o más partidas de un mismo estado financiero o de combinaciones de partidas de estados financieros; la metodología permite clasificar a los bancos en términos de su desempeño en dos grupos (satisfactorios e insatisfactorio) y dentro de cada grupo en bancos definidos como AAA, AA-Plus, AA, A y O.

Para su ejecución operativa se necesita identificar las variables que van a ser consideradas, la asignación de los códigos a los indicadores dependiendo de sus características y finalmente los resultados obtenidos de diez de los indicadores principales por banco, dos indicadores por cada categoría de evaluación.

El desempeño de cada banco se mide en base a la valoración que va de uno (1) a cinco (5) puntos, o su equivalente alfabético que va desde AAA, AA-Plus, $A A, A$ y $O$, significando cada uno lo siguiente:

AAA (5): Instituciones sanas financieramente.

AA-Plus (4): básicamente saludables, aunque pueda reflejar debilidades moderadas susceptibles de corregir.

AA (3): las instituciones ubicadas en esta categoría se caracterizan por tener problemas financieros, operacionales o de cumplimiento de las normas.

A (2): muestran excesivas debilidades en los activos o una combinación de limitaciones que generan resultados menos que satisfactorios, por lo que existen condiciones potenciales de colapso.

O (1): alta posibilidad de quiebra, requiriendo ayuda inmediata de sus accionistas.

Para la calificación de riesgo se identifica diez indicadores seleccionados (dos por categoría de estudio) Ver Tabla 1.

El procedimiento empleado para llegar a la calificación de riesgo, consiste en asignarle un puntaje a cada califica- 


\section{Tabla 1}

Indicadores de evaluación de la actividad finaciera bancaria

\begin{tabular}{|c|c|c|c|c|c|}
\hline \multirow[b]{2}{*}{ CAMEL INDICES } & \multicolumn{5}{|c|}{ Cualidad } \\
\hline & $\begin{array}{l}\text { Tendencia } \\
\text { Deseable }\end{array}$ & Muy Buena & Bueno & Regular & Malo \\
\hline
\end{tabular}

\section{Patrimonio}

$A=($ Patr+Gest.Op)/Act. T

$\mathrm{B}=$ Otros Act/Patr.

\section{Calidad de Activos}

$\mathrm{C}=$ Otros Act/Act.T.

$\mathrm{D}=$ Act.Improd/Act.T

\section{Gestión Operativa}

$\mathrm{E}=$ Marg. Fin/ Act. Prom.

Alta

Baja

$F=($ Gtos. $P+O p) /$

Act Prod Prom.

\section{Rentabilidad}

$\mathrm{G}=$ Util.N/ Act.Prom.

$\mathrm{H}=$ Util. N./Patr. Prom.

Liquidez

I= (Disp- Rend. Cobrar $)$

Capt.

$J=($ Disp. - Efect de Cobro

inm) + Inv

Temp)/(Capt-GtosxPag

Fuente: SUDEBAN (1998).

Tabla 2

Calificación de riesgo según metodología SUDEBAN

\begin{tabular}{cccc}
\hline Código & Categoría & Calificación & Clasificación \\
\hline 5 & AAA & AAA $\geq 70$ puntos & Satisfactorio \\
4 & AAPLUS & $60 \leq \mathrm{AAPlus} \leq 69$ & \\
3 & $\mathrm{AA}$ & $50 \leq \mathrm{AA}<60$ & Insatisfactorio \\
2 & $\mathrm{~A}$ & $30 \leq \mathrm{A}<50$ & \\
1 & $\mathrm{O}$ & $\mathrm{O}<30$ & \\
\hline
\end{tabular}

Fuente: SUDEBAN (1998). 
ción según sea Muy buena (10), Buena (6) , Regular (3), y Malo (0).

Posteriormente se elabora el siguiente cuadro de ponderación para calificar el riesgo (Tabla 2).

La metodología será aplicada para determinar el riesgo que experimentan los bancos universales y verificar cual ha sido su evolución en el periodo de estudio.

\subsection{Determinación de los valores discriminantes}

Para realizar esta investigación se empleó la fórmula obtenida de la aplicación del análisis discriminante sobre los datos de la banca comercial venezolana en el periodo junio de 1987 diciembre de 1994, presentado en el proyecto de investigación "Evolución de la Banca Comercial Venezolana periodo 19871994 " ${ }^{1}$, el cual será uno de los enfoques considerados para el desarrollo de este trabajo. Para obtener la fórmula discriminante se utilizó el procedimiento presentado en el trabajo antes mencionado el cual se expresa a continuación:

En la consecución del Valor Discriminante, se empleó la técnica de análisis de regresión del paquete estadístico SAS para obtener aquellos indicadores que explicarán en mayor medida el comportamiento de la banca en general tomando como base el siguiente modelo de regresión:

$A i=b o+b 1 X i 1+b 2 X i 2+\ldots \ldots .+b n X i n+\mu:$
Donde:

Ai: Indice de Predicción de la observación de un Banco Intervenido o Bancos no Intervenidos (saneados), dependiendo del valor que asuma Ai. (Variable Dependiente)

Xin: Variable Independiente representada por los Indicadores Financieros seleccionados en el método CAMEL.

bo: Coeficiente de Regresión o Intercepto que indica el valor promedio que tomará $\mathrm{Ai}$, suponiendo que los valores de las variables $(\mathrm{Xn})$ son cero.

b1.b2...bn: Coeficiente de Regresión que acompaña a la variables independiente Xin, los cuales explica la variación que asumirá la variable dependiente (Ai) al cambiar una o todas las variables independientes (Xin).

$\mu$ : Variable aleatoria, no observable que asume valores tanto positivos como negativos (Error estadístico).

Luego de efectuado el análisis, se procedió a utilizar el factor $\mathrm{R}^{2}$, el cual toma valores entre 0 y 1 significando que aquellas variables independiente que posean un valor cercano a 1 tendrían un mayor nivel de explicación en forma conjunta, como elemento de discriminación se escogieron, para el análisis de razones, aquellos índices con un $\mathrm{R}^{2}$ superior a 0.60 dando como resultado la selección de 17 indicadores los cuales son:

\section{Evaluación de Patrimonio}

Ep1 Patrimonio/Activo total. Ep3 Otros Activos/ Patrimonio.

$1 \quad$ Proyecto de Investigación, "Evolución de la Banca Comercial Venezolana periodo 1987-1994. Centro de Estudio de la Empresa, FCES. La Universidad del Zulia. Proyecto subvencionado por el CONDES. Investigador responsable Anibal Mujica. 
Ep4 Activo Improductivo/

Patrimonio.

Ep5 Total Pasivo/Patrimonio

Calidad de Activo.

Ca1 otros Activos/Activo Total.

Ca2 Activo Fijo/Activo Total.

Ca3 Activo Productivo/Activo Total

Ca4 Activo Improductivo/Activo

Total.

\section{Rentabilidad.}

R1 Ingresos Financieros ${ }^{*}$ Cartera de Crédito/Cartera de Crédito promedio.

R2 Ingresos Financieros ${ }^{*}$ Cartera de Inversión/Cartera de Inversión promedio.

R5 Margen Financiero/Activo Promedio.

\section{Gestión Administrativa.}

Ga4 Egresos Financieros/Captaciones promedio

\section{Liquidez.}

L1 Cartera de Crédito/ Captaciones Totales.

L2 Cartera de Inversiones/ Captaciones Totales.

L3 Disponibilidad en Moneda Nacional/ Captaciones Totales.

L5 Depósitos en B.C.V. +Valores Emitidos por la Nación/Activo Total ${ }^{\star} 100$.

L6 Deposito en B.C.V+ Valores emitidos por la Nación/ Captaciones Totales+Obligaciones a la vista.

Se seleccionaron aquellos índices del método CAMEL cuyo parámetro $\mathrm{R}^{2}$ fuera igual o mayor a 0.70 eligiéndose los siguientes índices:

1.Ep3 Otros Activos/ Patrimonio.

2.Ep4 Activos Improductivo/Patrimonio.

3.Ca1 Otros Activos/Activo Total.

4.Ca2 Activo Fijo/Activo Total.
5.Ca4 Activo Improductivo/Activo Total.

6.R1 Ingresos Financieros ${ }^{*}$ Cartera de Crédito/Cartera de Crédito promedio.

7.R2 Ingresos Financieros ${ }^{*}$ Cartera de Inversión/Cartera de Inversión promedio

8.Ga4 Egresos Financieros/Captaciones promedio

9. L2 Cartera de Inversiones/ Captaciones Totales.

Luego se escogieron dos grupos de Bancos tomando aquellos Bancos con mayor importancia:

Banco Intervenidos conformados por seis Entidades: Latino, Venezuela, Maracaibo, Consolidado, Metropolitano y República.

Bancos no Intervenidos igualmente conformados por seis entidades: Provincial, Mercantil, Lara, Caribe, Caracas y Venezolano de Crédito; siendo denominados los primeros Grupo 0 y los segundos Grupo 1. Posteriormente, se conformó una matriz especificando los nueve indicadores seleccionados cuyos valores estaban determinados por los promedios de las partidas financieras del período junio de 1989 a diciembre de 1993 de los estados financieros consolidados por agrupación; dicha matriz se ingresó en el paquete estadístico S.A.S utilizando la siguiente ecuación discriminante:

$\mathrm{Z}=\mathrm{b} 1 \mathrm{X} 1+\mathrm{b} 2 \mathrm{X} 2+$ $+b n X n$

Donde:

Z: Valor Discriminante o Indice de Predicción. (entre los grupos intervenidos $y$ no intervenidos).

X1;X2.......Xn: Variables Predictoras. (Indicadores Seleccionados para el modelo. 
b1;b2.........bn: Parámetros a estimar.

El paquete estadístico S.A.S, además de realizar el análisis discriminante, previamente efectuó un nuevo análisis de regresión donde seleccionó sólo tres indicadores de los nueve ya preseleccionados, los cuales se mencionan a continuación:

1.Ep3 Otros Activos/ Patrimonio.

2.Ep4 Activos Improductivo/ Patrimonio.

3.Ca1 Otros Activos/Activo Total. De dicha aplicación se obtuvo la siguiente fórmula discriminante:

(Y'EP3EP3PROMEDIO)+(Y'EP4*EP4PROMEDIO)+(Y'CA1*CA1PROMEDIO) (MUJICA Y CASTELLANO, 1999)

Donde:

Y'EP3= Resta entre el grupo O y 1 , de los valores de la fórmula para el indicador Otros Activos/ Patrimonio.

Y'EP4= Resta entre el grupo O y 1 , de los valores de la fórmula para el indicador Activos Improductivo/Patrimonio.

$Y^{\prime} \mathrm{CA} 1=$ Resta entre el grupo $O$ y 1 , de los valores de la fórmula para el indicador Otros Activos/Activo Total.

EP3PROMEDIO= Promedio del indicador Otros Activos/ Patrimonio con datos consolidados para el periodo de estudio.

EP4PROMEDIO= Promedio del in dicador Activos Improductivo/Patrimonio con datos consolidados para el periodo de estudio.

CA1PROMEDIO= Promedio del in dicador Otros Activos/Activo Total con datos consolidados para el periodo de estudio.
En base al cual se obtuvieron los siguientes valores discriminantes (Tabla 3 ).

Tomando como base la fórmula antes presentada, se sustituyo sus valores con datos de los indicadores seleccionados para el periodo de estudio junio 1996 -octubre 1998 de los bancos: Banesco, Caracas, Mercantil, Provincial, Venezuela, Venezolano de Crédito y Unión, obteniéndose el siguiente valor discriminante:

\section{Valor Discriminante: 42,68905265}

Por consiguiente, aquellos bancos que poseen valores inferiores al valor discriminante se considerarán bancos con salud financiera. Y los bancos cuyos valores superen el valor Discriminante serán denominados o considerados bancos con problemas financieros. Esto, dado que el valor discriminante se origina de índices cuyos valores deben ser bajos ya que sus numeradores son activos improductivos.

\section{Determinación de los factores que influyen en el riesgo asociado a la banca universal venezolana}

Para conocer el comportamiento del entorno al cual se enfrenta o con el cual interactúa la banca universal y el sector bancario en general, se elaboró el

\begin{tabular}{ccc}
\multicolumn{3}{c}{ Tabla 3 } \\
Valores discriminantes \\
\multicolumn{3}{c}{ Banca } \\
Gomercial Venezolana \\
\hline Grupo 0 & Grupo 1 & Y' (Prima) \\
\hline 92,8000 & 55,3813 & 37,4187 \\
22,0801 & 11,3073 & 10,7728 \\
2220 & 1516 & 704,00 \\
\hline
\end{tabular}

Fuente: Mujica et al (1997). 
siguiente estudio: calidad de la gerencia macroeconómica y política, descripción del sector, perspectivas del negocio y el entorno económico, para finalmente observar el efecto del entorno en la banca universal.

\subsection{Calidad de la gerencia macroeconómica y política}

El comportamiento de las variables macroeconómicas fundamentales, revelan el desenvolvimiento de la Banca Universal, para los períodos objeto de estudio. La calidad de la gerencia macroeconómica y política en Venezuela a partir del año 1999 comienza a avizorar señales de estabilidad, aún con las perspectivas de caída del Producto Interno Bruto (PIB).

Es notable el ambiente de inestabilidad política que se percibe en el país, desde el año de 1998, teniendo como aspecto más resaltante el proceso constituyente, cuya carta magna entró en vigencia recientemente, esperando la redacción de las leyes necesarias para su aplicación.

Las expectativas de inflación definitivamente son inferiores para 1999 ubicándose dentro de los parámetros establecidos por el gobierno $(20,0 \%$ de inflación según fuente de la Oficina Central de Información OCEI). Esta situación conlleva a crear un clima de confianza y credibilidad para la gerencia en Venezuela, aún observándose indefiniciones a nivel económico.

Destacan también dentro del contexto del sistema político venezolano, los acuerdos y alianzas del actual gobierno del Sr. Hugo Chavéz, con diferentes sectores económicos y políticos a nivel nacional e internacional y dado a su fuerza emanada del mismo proceso constituyente.

Dentro de este marco, Venezuela tiene por delante tal vez dos años de recuperación del PIB, aún observándose las secuelas que trae consigo en la economía nacional la caída en los niveles del empleo y del consumo, ocurrida a partir de 1998, situación esta analizada, por un conjunto de personalidades entre las cuales destacan, Hugo Faría (1999), Antonio Francés (1999), entre otros, los cuales prevén continuidad en la recesión de la economía del país. En este sentido, las industrias previeron una disminución de la demanda y para ello, activaron planes de contracción en su producción. Lo mismo ocurre en el mercado inmobiliario y en el sector de la construcción. La demanda de oficinas, locales y viviendas en todo el país, especialmente en las zonas impactadas por la industria petrolera destaca tal situación.

Un aspecto de singular importancia lo constituye el manejo del índice de inflación el cual se mantiene dentro de las perspectivas del gobierno, dada la fuerte caída del consumo y del control que mantiene el ente monetario sobre la liquidez monetaria.

Por otra parte, el evento denominado VISIÓN 99, realizado por el Instituto de Estudios Superiores de Administración (IESA) el cual se realizó el 10 de febrero de 1999, donde participaron importantes voceros del ámbito económico del país, destaca las perspectivas de caída del crecimiento de la economía venezolana, donde el producto interno bruto (PIB), experimentaría tasas cercanas al 6 ó 7 por ciento en 1999, observando que para el 
año 2000 se espera un incremento en el PIB, dado el fuerte impulso del gasto público a la economía con un presupuesto de 17,8 millardos de bolívares. El cual generará un incremento en el empleo y un aumento en el consumo, produciendo nuevos empleos en el corto plazo, sobre todo empleos de mano de obra semicalificada; en el sector petrolero se mantendrá la contracción de la actividad petrolera, la cual ha sido y continuara siendo el motor que impulsa el resto de las actividades económicas.

Dentro de este orden de ideas, Hugo Faría (1999) no observa en los anuncios económicos del gobierno la voluntad de transformar al país hacia una dirección donde se genere estabilidad y crecimiento económico; en sus comentarios expresa que para generar el equilibrio económico el actual gobierno incrementa los impuestos y no propicia las privatizaciones, retrasando la privatización de la industria del aluminio. Por consiguiente, en el mediano plazo las perspectivas son menos optimistas; ello obedece al esquema de funcionamiento de la economía venezolana la cual en los próximos tres años girará en torno a tres aspectos fundamentales: la presión social, el reparto de la renta petrolera y el reparto del poder. Destaca nuevamente el rol del gobierno en el manejo de las políticas económicas que aseguren con programas de largo plazo la estructura de la economía venezolana, a fin de permitir a los gerentes del nuevo milenio contar con herramientas para establecer programas estructurales por sectores, en contraste con las decisiones cortoplacistas que se ven obligados a tomar los gerentes venezolanos actualmente.
En resumen, el año 2000 será un año de crecimiento económico, esta situación debe ser manejada con cautela dentro de una economía que viene de enfrentar una severa recesión. El punto de fundamental importancia, radica en la incertidumbre política que afectará las decisiones del gobierno en cuanto a la paridad cambiaria, la cual incide sobre los niveles de inflación, creando expectativas en el flujo externo de capitales, originando salidas de los mismos. En conclusión los continuos enfrentamientos entre los diferentes sectores del país, en el largo plazo afectará la planificación estratégica gerencial.

\subsection{Descripción del sector}

La Banca Universal, dentro del sector financiero, representa al subsector más innovador de la banca en general, ello por su reciente creación por la ley general de bancos publicada en Gaceta Oficial No 4641 de noviembre de 1993; la banca universal cumple funciones diversas en la economía como la de inversión, préstamos hipotecarios, intermediación financiera en general. Actualmente poseen un importante porcentaje de los depósitos totales del sector e igualmente un porcentaje importante de sus acciones se encuentran en manos foráneas; todo esto originado posterior al proceso de crisis financiera, en general la banca universal sufre un proceso de reestructuración fundamentado en asociaciones estratégicas y fusiones.

\subsection{Perspectivas del negocio}

En el año de 1999, se produjo una fuerte caída del PIB en el orden del 7,2 por ciento originado por la disminución de 
los precios petroleros y por las crisis internacionales, igualmente por la inestabilidad política causada por los continuos procesos electorales y por el proceso constituyente. Para el año 2000 se espera un crecimiento de la economía venezolana, aunque de poca magnitud como secuela del fuerte gasto público; esto repercutirá en el negocio bancario, obligando a las instituciones financieras a delinear planes para posicionarse en el mercado a futuro sobre todo tratando de diferenciar el producto a través de campañas publicitarias y promociones o a través de estrategias de líder en costos, realizando fusiones y asociaciones en momentos que la banca observa índices aún favorables; igualmente el sector buscará reducir sus costos por agencia, disminuyendo el número de agencias.

\subsection{Entorno económico}

Los acuerdos del gobierno con la banca, para disminuir el diferencial entre la tasa pasiva y la tasa activa (incrementando la tasa pasiva en un punto porcentual y disminuyendo la tasa activa en dos puntos), determinan un enfoque favorable de relaciones entre el sector bancario y el actual gobierno; igualmente el proceso de reactivación económica iniciado principalmente en el sector construcción y la puesta en marcha del programa de carro familiar, los cuales estimularán el otorgamiento de créditos y por lo tanto la intermediación financiera. De igual forma la recuperación coyuntural de los precios del petróleo, reducirá el déficit proyectado para el año 2000 y por lo tanto la depresión de la economía.

Adicionalmente el incremento del encaje legal a un $17 \%$, ha originado un movimiento a favor de las fusiones en el sector sobre todo en estos momentos en que las cifras de sus cuentas reflejan índices positivos, incrementándose la sinergia y asociaciones estratégicas entre instituciones financieras locales e internacionales. Basándose en la influencia del entorno que surge del análisis anteriormente realizado se pretende determinar su efecto en la situación financiera de la banca.

\section{Efecto de las políticas económicas en el riesgo bancario (evolución del riesgo sistemático)}

Para determinar el efecto del entorno económico-político del país sobre la situación financiera de la banca universal se calculará la elasticidad precio de la demanda ${ }^{2}$ de las acciones del sector; para su consecución se utilizará una proxy (Variable que mide indirectamente a otra variable la cual es difícil de medir o no cuenta con indicadores validos), de la elasticidad precio de la demanda, siendo esta el coeficiente de determinación entre el índice de capitalización bursátil de la Bolsa de Caracas a cierre de mes y el precio de cierre de las acciones de los bancos objeto de estudio. Se analizará el coeficiente de determinación $\left(r^{2}\right)$ de la siguiente forma: 
- Un $\left(\mathrm{r}^{2}\right)$ con tendencia 1 implica que la elasticidad precio de la demanda (EPD) es elástica $(n>1)$ por lo tanto las perspectivas del valor de la acción y de la empresa, depende de la situación del país.

- Un $\left(\mathrm{r}^{2}\right)$ con tendencia a 0.5 implica una tendencia difícil de predecir por lo tanto su interpretación es indiferente, elasticidad unitaria $(n=1)$.

- El $\left(r^{2}\right)$ con tendencia hacia 0 demuestra una elasticidad precio de la demanda de la acción inelástica $(\mathrm{n}<1)$ por lo tanto las perspectivas del valor de la acción y de la empresa no depende de la situación económica y política del país.

Posteriormente se elaboró un modelo multivariables de regresión simple suponiendo un comportamiento normal de la variable, dicho modelo es como sigue: $\mathbf{l b c}_{\mathbf{t}}=\beta_{1}+\beta_{2} \mathrm{Me}_{\mathbf{t}}+\beta_{3} \mathrm{Pr}_{\mathbf{t}}-{ }_{4} \mathrm{Ve}_{\mathbf{t}}+\varepsilon \mathrm{t}$ Donde:

Ibc: Indice de Capitalización al cierre de mes (Bolsa de Caracas). En el periodo t.

Me: Precio de Cierre Mensual de la Acción bancos Mercantil. En el periodo t.

Pr: Precio de Cierre Mensual de la Acción bancos Provincial. En el periodo t.

Ve: Precio de Cierre Mensual de la Acción bancos Venezuela. En el periodo t.

$\beta_{1}$ : Intercepto (valor del índice de capitalización cuando el precio de cierre de las acciones seleccionadas son iguales a cero).

$\beta_{2} \ldots . . . \beta_{8}$ : coeficiente de regresión, $\varepsilon$ : Término de error.

Esperando que los coeficientes se comporten como sigue

$$
\text { (+) } \beta_{1},(+) \beta_{2},(+) \beta_{3},(-) \beta_{4}
$$

Empleando Igualmente como criterio de selección de los bancos, el poseer información completa de sus cotizaciones, en otras palabras que coticen periódicamente en la Bolsa de Caracas.

- Número de Variables: 4

- Número de Observaciones: 36

- Grados de Libertad: 32

- Data: 31/01/96 30/12/98

- Nivel de Significación del 5\%

- Programa: Econometric Views 3 Student Version. En la Tabla 4 pueden verse los resultados del modelo de regresión.

Dentro de este marco, se realizará de este modelo los respectivos análisis Estadístico:

- Análisis Estadístico: Se mantiene un nivel de explicación significativo de 0.86 al $5 \%$, es decir el comportamiento de la variable dependiente es explicado por las variables independientes en muy buena medida, por ello el Prob(F-statistic) tiende a 0.00 , la multicolinealidad no existe, dado las tendencias de la Prob TStatistic en todos los casos, es decir no existe correlación entre las variables independientes; observándose la existencia de correlación serial positiva (Durbin - Watson no tendiente a 2) es decir que se viola el supuesto de que el error de los datos son independientes dentro del modelo de regresión lineal, los signos esperados para los coeficientes de regresión se cumple, dando las conclusiones de ello en el siguiente análisis, En los correlogramas se observa una disminución en la autocorrelación y sobre todo en los 


\section{Tabla 4 \\ Resultado Modelo de Regresión}

LS // Dependent Variable is ICB

Date: 06/14/01 Time: 18:28

Sample: 1996:01 1998:12

Included observations: 36

$\begin{array}{lrlrl}\text { Variable } & \text { Coefficient } & \text { Std. Error } & \text { T-Statistic } & \text { Prob. } \\ & & & & \\ \text { MERCANTIL } & 9.919753 & 1.892571 & 5.241417 & 0.0000 \\ \text { PROVINCIAL } & 7.289288 & 1.585518 & 4.597419 & 0.0001 \\ \text { VENEZUELA } & -0.357198 & 0.114600 & -3.116913 & 0.0038 \\ \text { C } & 1929.112 & 400.4284 & 4.817621 & 0.0000 \\ & & & \\ \text { R-squared } & 0.862643 & \text { Mean dependent var } & 5976.231 \\ \text { Adjusted R-squared } & 0.849766 & \text { S.D. dependent var } & 2181.330 \\ \text { S.E. of regression } & 845.4833 & \text { Akaike info criterion } & 13.58426 \\ \text { Sum squared resid } & 22874942 & \text { Schwartz criterion } & 13.76020 \\ \text { Log likelihood } & -291.5984 & \text { F-statistic } & 66.99013 \\ \text { Durbin-Watson stat } & 0.716305 & \text { Prob(F-statistic) } & 0.000000\end{array}$

rezagos después del quinto año sus valores están por debajo del 0.5 lo que se puede suponer la existencia de no estacionariedad, es decir las variables tanto independiente $\mathrm{y}$ dependiente tienen un comportamiento estacionario sobre el cual se puede hacer proyecciones o bien determinar comportamientos históricos. El test de White de heteroscedasticidad muestra la existencia de heteroscedasticidad del mismo por lo tanto se confirma el supuesto del comportamiento no constante del error. Se hace evidentes las debilidades del Modelo por la existencia de Correlación Serial y heteroscedasticidad.

- Análisis Económico: Dado que el modelo se ajusta a ciertos supuestos econométricos y consientes de sus debilidades, se procederá a realizar el

análisis económico. En base a ello se puede afirmar, tomando el $R^{2}$ que es igual a 0.86 , que la situación financiera de la banca es elástica ante la situaciones del entorno político económico; en otras palabras se puede inferir que la banca en el periodo de estudio se ha hecho elástica o bien es influida por la coyuntura de la política y la economía en el país. Adicionalmente los bancos que más contribuyen a la formación del ICB son los bancos más grandes en depósitos, y de capitales extranjeros (Bilbao Vizcaya: Provincial, Santander: Venezuela y Mercantil) es decir los bancos más importantes del país (es de hacer notar que el ICB se explica en buen porcentaje por si mismo dado que su intercepto (1929.112) es alto con respecto a su promedio (5976.231). 
En general el modelo se visualiza como sigue:

$\mathrm{lbc}=2241.843+6.552641 \mathrm{Me}+$ 8.392211Pr $-0.396731 \mathrm{Ve}+\varepsilon$

En base a este modelo puede inferirse, dada la fuerza explicativa que tiene el mismo, que el sector (Banca Universal) está incrementando su riesgo sistemático (se hace más dependiente del entorno macroeconómico).

\section{Aplicación del análisis discriminante}

Para la aplicación y análisis de los valores discriminantes se consideró el origen de los índices que la conforman, los cuales según la metodología de la Su- perintendencia de Bancos y Otras Instituciones Financieras (SUDEBAN), deben tener valores bajos (sin especificar un indicador deseado); por lo tanto el valor discriminante para bancos saludables debe ser bajo (menos a 42.3566) por ello en el Gráfico 1, se observa como el valor discriminante divide a las instituciones en bancos sanos financieramente y bancos con problemas financieros. De allí pues, que la mayoría de las entidades se encuentre por encima del valor, dentro del periodo de estudio notándose como sólo los bancos Venezolano de Crédito y Venezuela estuvieron por debajo del valor discriminante es decir con salud financiera, igualmente los Bancos Unión, Caracas y Banesco, se sitúan en todo el periodo de es-

\section{Gráfico 1 \\ Valores discriminantes Banca Universal Venezolana Período 1996-1998}

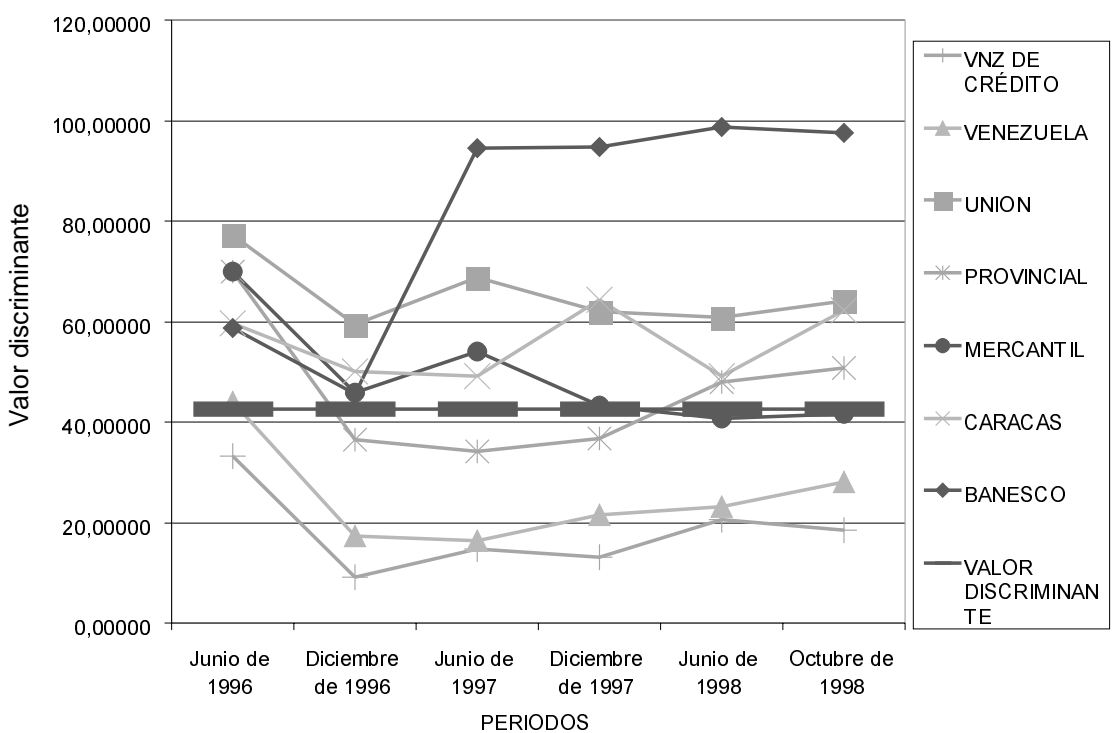

Fuente: Cálculos propios. 
tudio por encima del valor discriminante, el cual evidencia sus problemas financieros; Provincial y Mercantil poseen un comportamiento irregular especialmente Provincial cuyo valor tiende a mejorar entre los años 1996 y 1997 para nuevamente empeorar en el siguiente año, en general la tendencia de la muestra es hacia un leve incremento de sus respectivos valores discriminantes en los últimos periodos, observándose la fuerte tendencia alcista del banco Banesco para el periodo junio de 1997, para luego casi mantenerse.

\section{Determinación del riesgo asociado a la banca universal venezolana}

Teniendo como premisa que los bancos con desempeño satisfactorio se ubican en la categoría AAA y AAPlus, y los bancos con desempeño insatisfactorio en la categorías $A A, A$ y $O$; se obtiene como conclusión que el comportamiento de los bancos ha sido en general irregular, por ejemplo bancos como Venezolano de Crédito y Venezuela comenzaron con un desempeño satisfactorio (año 1996) para pasar en el año 1997 a insatisfactorio, culminando el periodo de estudio con desempeño satisfactorio. Caso contrario se observa en bancos como Banesco, Caracas y Mercantil, cuyo desempeño fue insatisfactorio para el periodo; el comportamiento del Unión ha sido alterno pasando de satisfactorio a insatisfactorio igual al del Provincial, el único banco que mantuvo su desempeño satisfactorio durante todo el periodo es el Banco de Venezuela.
En general el comportamiento de la muestra de la banca universal refleja un leve repunte del riesgo entre el periodo junio y diciembre de 1996 (ver Gráfico 2), debido a las secuelas de la debacle financiera aún permanentes en el sector. El riesgo observa un comportamiento hacia la baja desde diciembre de 1996 hasta enero de 1997, para posteriormente permanecer casi constante (Ver Gráfico 2).

\section{Factores externos e internos que afectan el comportamiento del riesgo bancario}

Para determinar los factores internos que afectan el comportamiento del riesgo en la banca universal, se procedió a comparar el comportamiento del valor discriminante con el comportamiento de la calificación del riesgo según la Metodología de la Superintendencia de Bancos y Otras Instituciones Financieras (ver Gráficos 1 y 2) puede observarse, que existe cierta relación entre el comportamiento de la variable riesgo y del valor discriminante sobre todo en el año de 1996, reflejándose posteriormente tendencias estabilizadoras, (aunque en la aplicación de la metodología de la SUDEBAN en el periodo octubre de 1998 no puede analizarse dado que en los estados financieros suministrados por la Superintendencia de Bancos faltaban algunas partidas lo cual imposibilita el cálculo del riesgo exacto para ese periodo).

Por consiguiente, se puede determinar qué factores internos del sector afectan el valor asumido por el riesgo, tomando en cuenta que el valor discriminante fue calculado utilizando para ello 


\section{Gráfico 2 \\ Calificación de riego Banca Universal Venezolana Período 1996-1998}

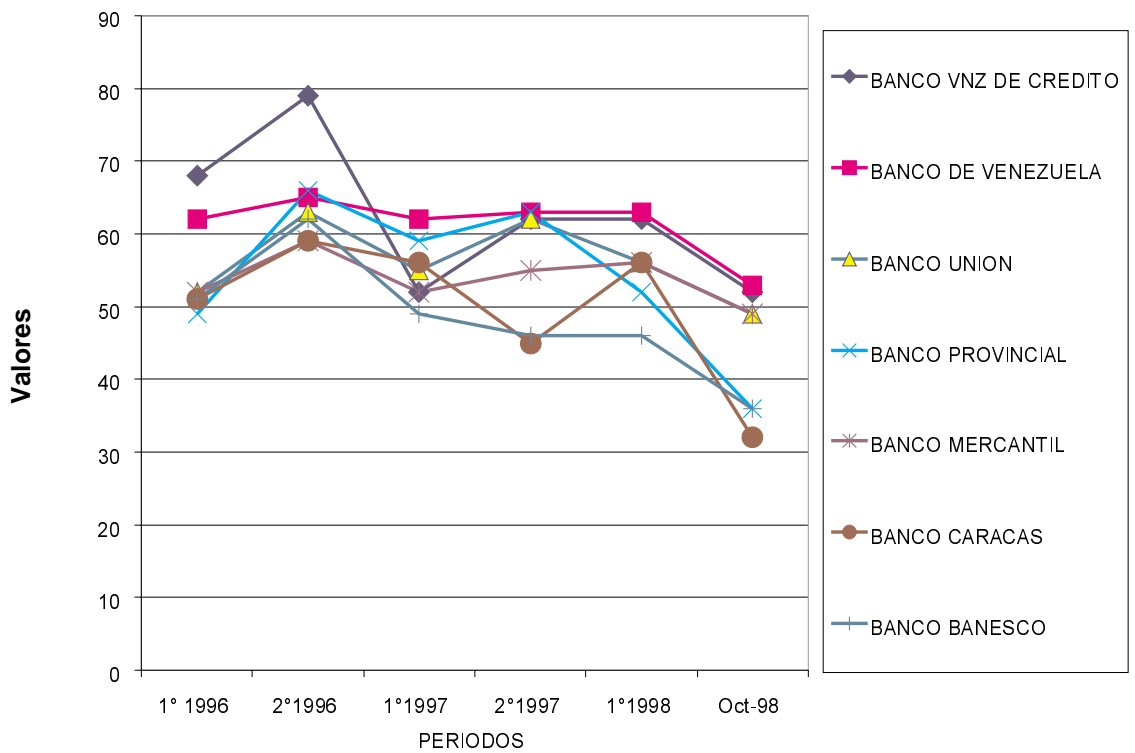

Fuente: Cálculos propios.

sólo tres indicadores de los existentes en la metodología CAMEL:

- Otros Activos / Activo Total (Calidad de los Activos ca1)

- Otros Activos / Patrimonio (Evaluación del Patrimonio ep3)

- Activos Improductivos / Patrimonio (Evaluación del Patrimonio ep4)

Se observa como estos indicadores están formados por partidas como otros activos y activos improductivos (factor numerador) los cuales son considerados "activos improductivos para un banco" dado que no representan rentabilidad para la institución, además de ser activos que no se incluyen dentro de las operaciones propias de la función primordial de la banca, la cual se refiere a la intermediación financiera. Por ello mientras menor sea la proporción de estos activos con respecto al activo total y al patrimonio, menor será el valor del indicador, por ello al analizar el valor discriminante (que posee la bondad de reunir en su conjunto los tres indicadores ya mencionados), tenemos que mientras menor sea su valor mejor será la situación financiera de la institución en estudio. En este sentido se comprende, que sobre todo en el año de 1996 mientras menor fue el valor discriminante la calificación del riesgo mejoró considerablemente en todos los bancos de la muestra, igualmente se observa como los bancos Venezolano de Crédito 
y Venezuela, quienes mantienen en todo el periodo de estudio las mejores calificaciones de riesgo, poseen los menores coeficientes en los valores discriminantes; de hecho si el análisis se diera banco por banco la coincidencia entre dichos valores sería mayor pero, dado que el estudio se centra en el sector, se realizará su análisis en conjunto. La existencia de coincidencias se explica por la naturaleza del origen del valor discriminante conseguido, el cual se basa en partidas improductivas y no acordes con la intermediación financiera; cuestión que permite afirmar que en la banca universal, en el periodo de estudio se origina un proceso de desintermediación financiera el cual no es originado por una coyuntura de competencia entre instituciones del ramo, bien sea actual o potencial, (aseguradoras, fondos de pensiones, fondos mutuales, entre otros) si no por políticas internas de las instituciones que a su vez permite afirmar el hecho de la no existencia en el cálculo del valor discriminante de indicadores de rentabilidad y liquidez, dado su poca significación y contribución a dicho valor. Este proceso de desintermediación está llevando al sector a realizar operaciones no acordes con sus funciones, que elevan sus calificaciones de riesgo. En otras palabras se afirma que la fórmula discriminante utilizada en este estudio mide con cierta seguridad el riesgo asociado a dichas instituciones.

Analizando los coeficientes de correlación y determinación del sector se vislumbra que los factores del entorno negativos para su desenvolvimiento poseen un importante peso, dado que el sector se ha hecho elástico, es decir la elasticidad precio de la demanda es elástica, por lo tanto los vaivenes del entorno tienen alta influencia en el sector bancario. con respecto a otros, lo cual lleva a las instituciones bancarias a implementa políticas que orientan a sus organizaciones a ser menos dependientes de la situación político económica del país dado el proceso de recesión causado principalmente por la caída de los precios petroleros, cabe considerar, por otra parte, que para el año 2000 se espera una recuperación de la economía causado en parte por el fortalecimiento del sector construcción.

\section{Conclusiones}

A nivel del entorno, la banca universal se desenvuelve dentro de un ambiente hostil, dada la recesión económica del país y por la inestabilidad de los precios petroleros, aunado a la inestabilidad política por los continuos procesos electorales los cuales incrementaron la crisis existente en los últimos años del periodo de estudio, lo cual explica el comportamiento de la calificación del riesgo para los últimos periodos. Ello lleva a la banca universal a aplicar políticas y estrategias, bien para diferenciar el producto vía campaña publicitaria, o bien siendo líderes en costos a través de procesos de fusiones entre entidades en momentos en que sus acciones reflejan estabilidad en sus situaciones financieras, para hacer que sus finanzas no se encuentren influenciadas por la situación política-económica del país, dado que su elasticidad precio de la demanda tiende a ser elástica, por lo tanto su situación financiera depende más del entorno político económico del país, incrementando su riesgo sistemático. 
El riesgo asociado a la Banca Universal Venezolana durante el periodo 1996-1998, ha observado un comportamiento tanto hacia la baja como hacia la alta, notándose tendencias alcistas hasta el segundo periodo del año de 1996, para luego disminuir en el primer periodo de 1997, entrando en los periodos segundo de 1997 y primero de 1998 en comportamientos difíciles de interpretar en forma grupal, lo cual se puede interpretar como el cambio de políticas internas de cada institución originado por la coyuntural situación de la economía venezolana y su repercusión en el sector. En este sentido, analizando el riesgo de mercado y elaborando una comparación entre los valores del análisis discriminante y la calificación de riesgo, se obtiene en los primeros periodos del análisis, casi un comportamiento similar, experimentando tanto la calificación de riesgo como el valor discriminante, las mismas tendencias, pero en forma inversa, en los periodos subsiguientes del estudio, pudiendo no obstante comprobar que dicho valor discriminante mide internamente el riesgo de mercado de la banca universal para el periodo de estudio; esto indica que dado que el valor está compuesto por los indicadores otros activos/activo total (ca1), otros activos/patrimonio (ep3) y activos improductivos/patrimonio (ep4) el porcentaje de las partidas otros activos y activos improductivos con respecto al patrimonio y al activo total influyen enormemente en la calificación del riesgo, debido a que estos indicadores están formados por partidas consideradas como activos improductivos dado que no representan rentabilidad para la institución, por lo tanto no se corresponden a las operaciones propias de intermediación; concluyéndose que el sector se encuentra en un proceso de desintermediación financiera no originado por competencias actuales o potenciales de otras instituciones, sino por políticas internas que han apartado a la banca de sus funciones propias.

Sucede pues, que tomando en cuenta que existe una correspondencia entre el valor discriminante y la calificación del riesgo, se puede afirmar que la tendencia general de la muestra seleccionada de la banca universal venezolana posee un comportamiento leve hacia el alza en el último periodo estudiado (octubre de 1998), el cual no se observa en la calificación del riesgo dado que en los estados financieros (suministrados por la superintendencia de bancos y otras instituciones financieras) poseían fallas en sus partidas, lo cual imposibilitó un cálculo veraz del riesgo para dicho periodo.

Con base en estas afirmaciones se demuestra la confiabilidad y seguridad de la fórmula discriminante utilizada en el estudio, la cual fue hasta cierto grado capaz de medir el riesgo al cual se enfrenta la Banca Universal, pudiendo ser utilizada para posteriores estudio en la construcción de modelos predictivos basados en estos tipos de activos y de indicadores, que pueden demostrar el cumplimiento de las funciones de la banca, disminuyendo el peso de los indicadores de rentabilidad y liquidez en este tipo de evaluaciones, debido a que estas por si solas no muestran el riesgo de la Banca Universal.

Es de hacer notar que aún con los severos problemas por los cuales el país ha pasado y sigue pasando, incrementando su calificación de riesgo país, la banca se ha mantenido aplicando estra- 
tegias para estabilizar sus calificaciones de riesgo de mercado, sobre todo instituciones como los Bancos Venezuela y $\mathrm{Ve}$ nezolano de Crédito, los cuales mantienen uniformes sus respectivas calificaciones de riesgo satisfactoriamente. Igualmente sus respectivos valores discriminantes se encuentran en todo el periodo de estudio por debajo del valor discriminante, explicado por sus bajos indicadores de activos improductivos/ patrimonio, otros activos/patrimonio y otros activos/activo total.

De la aplicación del modelo econométrico construido, se puede inferir que se ha originado un proceso de elasticidad entre el entorno político económico y la situación financiera del sector bancario, provocando un incremento del riesgo sistemático, haciendo al sector más dependiente de su entorno macroeconómico.

\section{Bibliografía Citada}

Faría, Hugo (1999). Perspectivas Económicas del año 1999. Comunicado del foro del IESA Visión 99, Caracas, Maracaibo, Valencia.

Francés, Antonio (1999). Posibles Consecuencias para Venezuela de la adopción de diferentes programas Económicos. Comunicado del foro del IESA Visión 99, Caracas, Maracaibo, Valencia.

López, Joaquín, Sebastián, Altina (1998). Gestión Bancaria Los Nuevos Retos en un
Entorno Global. Serie McGRAW-HILL de Management. Madrid. pp 252.

Mujica F., Aníbal; Castellano, Alberto (1999). Análisis de la Situación Financiera de la Banca Comercial periodo 1987- 1994. Revista de Ciencias Sociales. FCES-LUZ, Nueva Época. Vol. 5, .N 2. Maracaibo, Venezuela. Pp. 49-94.

Mujica F., Aníbal; Prieto, Nergio; Castellano, Alberto (1997). Evolución Financiera de la Banca Comercial Venezolana periodo 1987-1994. Documento, Centro de Estudio de la Empresa, FCES, LUZ. Maracaibo, Venezuela. Pp 305.

Ramírez, Douglas y Díaz, Carlos (1999). Un Modelo Explicativo del Proceso de Desintermediación Financiera en Chile. Universidad Católica del Norte. Presentado en el XVI Encuentro Nacional de Escuelas y Facultades de Administración y Economía (ENEFA 99) Universidad de Santiago de Chile. Pag 396-409.

Sabal, Jaime (1997). La Gerencia de Riesgo: Una Introducción al Tema. Debate lesa. Riesgo Financiero. Vol. 2. No. 3. Ene - Mar. pp 45.

Superintendencia de Bancos y otras Instituciones Financieras (SUDEBAN) (1998). Metodología para la Clasificación de Riesgo de los Bancos Comerciales y Universales según su Desempeño. Gerencia Técnica, Departamento de Estadística, SUDEBAN. Caracas. pp 27. 